\title{
Preparation of PDOT:PSS Transparent Conductive Film Using Ink-Jet Printing
}

\author{
Atsushi Nitta ${ }^{*}$, Kazuki Shimono ${ }^{2,3}$ \\ ${ }^{1}$ Department of Electronic Control Engineering, National Institute of Technology, Kagoshima College, Kirishima, \\ Japan \\ ${ }^{2}$ Advanced Mechanical and Electronic Systems Engineering, National Institute of Technology, Kagoshima \\ College, Kirishima, Japan \\ ${ }^{3}$ School of Materials Science, Japan Advanced Institute of Science and Technology, Ishikawa, Japan \\ Email: "nitta@kagoshima-ct.ac.jp
}

Received 4 November 2015; accepted 1 December 2015; published 4 December 2015

Copyright (C) 2015 by authors and Scientific Research Publishing Inc.

This work is licensed under the Creative Commons Attribution International License (CC BY).

http://creativecommons.org/licenses/by/4.0/

(c) (i) Open Access

\section{Abstract}

Flexible devices produced using organic materials have attracted the attention of many researchers. Important components of these flexible devices include transparent electrodes, which transmit visible light and possess conductivity. The present study improved the characteristics of a transparent conductive film that was made of poly(3, 4-ethylenedioxythiophene):poly(styrenesulfonate) (PEDOT:PSS), an organic conductive material, and that had been prepared using ink-jet printing. To improve the resistance value and visible light transmittance of the film, the film substrate was first cleaned with ultraviolet/ozone treatment, and then the film was annealed after it was deposited on the substrate and dipped into a polar solvent. Consequently, the resistance value of the thin film decreased. However, the surface state of the film changed according to the treatment method and affected its visible light transmittance. Thus, the surface state of the film substrate, the annealing temperature after film deposition, and the dipping treatment with a polar solvent influenced the characteristics of a thin film.

\section{Keywords}

Organic Electronics, Transparent Conductive Film, Ink-Jet Printing, PEDOT:PSS

\section{Introduction}

Flexible devices produced using organic materials have recently become a major focus of research interest [1]-[5]. Organic electroluminescence displays, organic thin-film solar cells, and organic transistors have been

${ }^{*}$ Corresponding author. 
actively studied. In these electronic devices, transparent electrodes, which transmit visible light and possess conductivity, are important components [6] [7]. At present, an indium tin oxide (ITO) thin film is often used as a material for a transparent conductive film. However, because an ITO thin film contains indium, a rare metal, depletion and insufficient supply of the resource have been concerns. Moreover, because an ITO thin film shows fragility to bending stress and because it is produced using the sputtering method, which requires high-temperature vacuum treatment, an ITO thin film cannot be used for developing flexible devices; instead, various materials, such as organic materials, metallic nanoparticles, and carbon nanotubes (CNT), have been studied [8]-[13]. In particular, organic materials are noteworthy because a film can be formed using a low-temperature process, and cheap devices can be manufactured by printing and coating [14] [15].

Device manufacturing technology using printing techniques is called printed electronics, and this is used for a wide range of product lines, including interconnection fabrication [16]-[18]. As the study of printed electronics advances, certain types of electronics equipment are expected to be produced using only this method [19] [20]. The advantages of printed electronics include minimized manufacturing costs, reduced environmental loads, and expansion of the area of a device.

If a practicable transparent conductive film can be produced using a simple and cheap method, such as a printing method, not only electrodes but also electronic apparatuses can be manufactured at low cost. However, to use printing technologies, a material must be prepared in the form of ink, and the prepared ink must be optimized. Moreover, the performance of an organic transparent conductive film must be improved; otherwise the film cannot be practically used.

In the present study, we focused on poly(3, 4-ethylenedioxythiophene):poly(styrenesulfonate) (PEDOT:PSS), an organic conductive material, as a substitute for ITO. We formed a film on a plastic film substrate using a commercially available ink-jet printer. Thus, a cheap and flexible transparent conductive film could be produced. In previous studies, we optimized ink by changing the types of additives and their mixing ratios and evaluated the conductivity and transparency of the obtained thin film. In the present study, to improve the characteristics of the thin film, the film substrate was cleaned with ultraviolet/ozone $\left(\mathrm{UV} / \mathrm{O}_{3}\right)$; the film was annealed after it was deposited on the substance; and the film was then dipped into a polar solvent. Subsequently, the effects of these treatments were examined.

\section{Experimental}

\subsection{Preparation of Ink Materials for a Transparent Conductive Film}

In the present study, CLEVIOS (Clevios $\left.{ }^{\mathrm{TM}} \mathrm{PH} 500\right)$ was used as PEDOT:PSS. Figure 1 shows the molecular structure of the PEDOT:PSS. The ratio of PEDOT to PSS was 1 to 2.5, the boiling point of the PEDOT:PSS was approximately $100^{\circ} \mathrm{C}$, the viscosity was below $25 \mathrm{~m}$ Pa-s, and the particle size was below $50 \mathrm{~nm}$. Because the nozzle diameter of a commercially available ink-jet printer is between several micrometers and tens of micrometers, the PEDOT:PSS could be used for the ink-jet method. However, because the viscosity of ink for an ink-jet printer is 5 - $15 \mathrm{~m} \mathrm{Pa-s,} \mathrm{it} \mathrm{was} \mathrm{important} \mathrm{to} \mathrm{reduce} \mathrm{the} \mathrm{viscosity} \mathrm{of} \mathrm{the} \mathrm{PEDOT:PSS} \mathrm{ink.} \mathrm{We} \mathrm{therefore}$

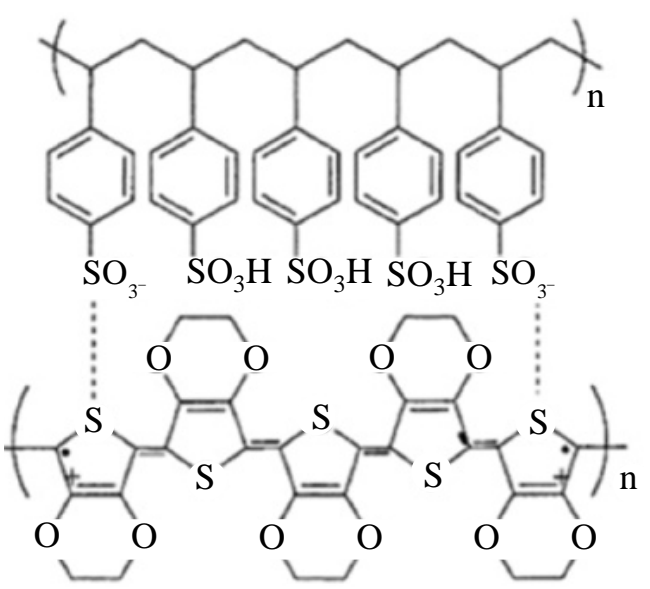

Figure 1. The molecular structure of the PEDOT:PSS. 
added ethanol to adjust the viscosity of the PEDOT:PSS ink and to improve the wettability of the substrate. Ethanol is a volatile liquid with a boiling point of $78^{\circ} \mathrm{C}$ and a viscosity of $1.078 \mathrm{~m} \mathrm{~Pa}$-s.

We added ethylene glycol to improve the conductivity of the PEDOT:PSS thin film. Ethylene glycol is a liquid with a boiling point of $198^{\circ} \mathrm{C}$ and a viscosity of $16.1 \mathrm{~m}$ Pa-s. The improvement in the conductivity by adding ethylene glycol is considered to be due to secondary doping and coupling of conductive regions [21]. Adding ethylene glycol reportedly removes insulative PSS and forms a conductive region consisting of conductive PEDOT [22]. We adopted the ink composition of PEDOT:PSS:ethanol:ethylene glycol $=70: 20: 10 \mathrm{wt} \%$, which exhibited the lowest resistance in previous studies [23].

\subsection{Preparation and Evaluation of a Thin Film}

To produce a PEDOT:PSS thin film, a piezo-type, pigment-based, ink-jet printer (PX-101; Seiko Epson CO.) was used, and, a heat-resistant transparent film (TeonexQ65-FA; Teijin DuPont Films Co, Ltd.) was used as a substrate. Q65-FA is a biaxially-oriented polypropylene naphthalate film possessing excellent thermal stability, chemical resistance, and transparency. The size of the film substrate is $30 \times 10 \mathrm{~mm}$. The surface of the film substrate was cleaned using a UV/O ${ }_{3}$ cleaning and modifying apparatus (ASM401N; AsumiGiken, Ltd). The cleaning time was $20 \mathrm{~min}$, and the UV radiation distance was $30 \mathrm{~mm}$. The output of a low-pressure mercury lamp was $40 \mathrm{~W}$. An additive was added to improve the viscosity and surface tension of the ink. However, the additive remained in the thin film as impurities after it was formed, and as a consequence of this residual additive, the resistance value of the thin film was considered to increase. In the drying process, if a thin film is annealed to evaporate the residual additive, the resistance value is expected to decrease. For drying, a constant-temperature drying oven (EOP-300B; As One Co, Ltd.) was used. The temperature was set at $80^{\circ} \mathrm{C}, 100^{\circ} \mathrm{C}$, or $150^{\circ} \mathrm{C}$, which is higher than the boiling point of ethanol. The thin film was dried for 5 min after each printing.

Drying a PEDOT:PSS thin film again after dipping it in a polar solvent reportedly facilitates the arrangement of PEDOT molecules, thereby improving the conductivity of the PEDOT:PSS thin film [24]. A polar solvent is a liquid consisting of polar molecules; these are molecules in which the center of gravity of a positive electric charge disagrees with that of a negative electric charge. In a polar molecule, an electric dipole exists spontaneously and permanently. The size of a crystal in which PEDOT molecules are arranged reportedly increases as the polarity of a polar molecule becomes stronger; consequently, the electric conductivity of a PEDOT:PSS thin film improves [24]. We used ethylene glycol as the polar solvent, and the PEDOT:PSS thin film was dried at $100^{\circ} \mathrm{C}$ for 60 min after dipping it in ethylene glycol.

We used a digital multi-meter (VOAC7521H; Iwatsu Electric Co, Ltd.) to evaluate the conductivity of the obtained thin film and a spectrophotometer (U-3900; Hitachi High-Technologies Co) to evaluate transparency. We used a microscope (VHX-1000; Keyence Co, Ltd.) to observe the surface.

\section{Results and Discussion}

\subsection{Differences in Resistance Value and Transmittance According to the Number of Printing Times}

When producing a transparent conductive film using the ink-jet method, the resistance value of the film is extremely high when the printing is not performed several times. In the present study, the printing was performed several times in order to reduce the resistance value of the film.

Figure 2 shows the changes in the resistance value and visible light transmittance according to the number of printing times. Printing up twice had a very high resistance value and the variation of the measured values was large. The resistance value of the thin film decreased as the number of printing times increased. The reason for this is probably that the printing patterns became dense and the contact area between PEDOT:PSS particles increased. However, visible light transmittance of the thin film decreased as the number of printing times increased. Because PEDOT:PSS is a dark blue liquid, the color remained in the obtained thin film. Visible light transmittance above $80 \%$, which is required for a transparent conductive film, could not be attained in printing six times or more. Therefore, the effect of the remaining color became more significant as the number of printing times increased. Multiple printing times is therefore effective in reducing the resistance value but has limitations when transparency is considered. The present study focused on thin films being printed three to five times while considering the resistance value and visible light transmittance. 


\subsection{Improvement in the Substrate Surface by UV/ $\mathrm{O}_{3}$ Cleaning}

To examine the effect of the substrate surface on the characteristics of a thin film, the substrate surface was cleaned with $\mathrm{UV} / \mathrm{O}_{3}$. Figure 3 shows a microscope image of the surface of a PEDOT:PSS thin film. As a result of $\mathrm{UV} / \mathrm{O}_{3}$ cleaning of the substrate surface, ink could be smoothly spread and the homogeneity of the thin film increased.

Figure 4 shows the changes in the resistance value due to $\mathrm{UV} / \mathrm{O}_{3}$ cleaning and the number of printing times.

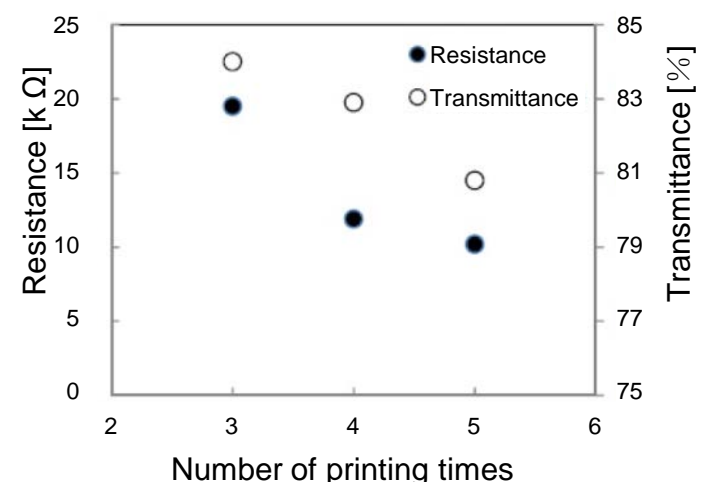

Figure 2. Changes in the resistance value and visible light transmittance according to the number of printing times.

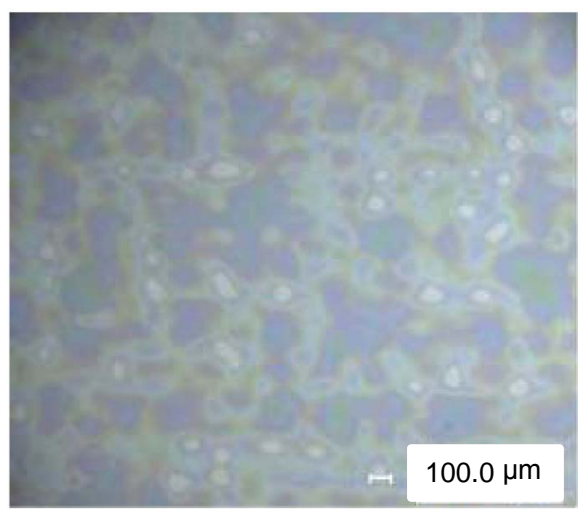

(a)

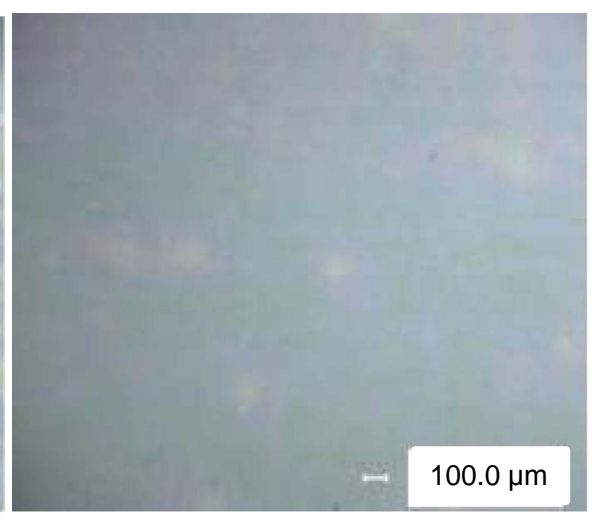

(b)

Figure 3. Microscope image of the surface of a PEDOT:PSS (5 times printing, $\times 100$ ). (a) Before cleaning; (b) After $\mathrm{UV} / \mathrm{O}_{3}$ cleaning.

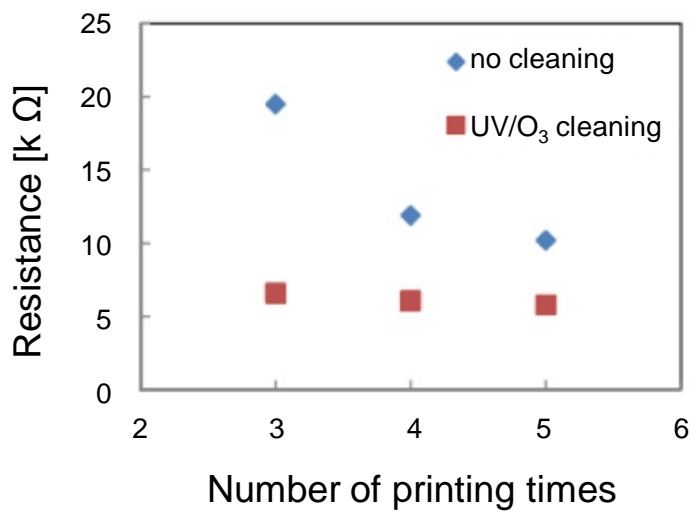

Figure 4. Dependence of the resistance value due to $\mathrm{UV} / \mathrm{O}_{3}$ cleaning and the number of printing times. 
As shown in this figure, the resistance value decreased when $\mathrm{UV} / \mathrm{O}_{3}$ cleaning was performed, possibly because UV cleaning and surface modification decreased the amount of impurities and increased the adhesion of a droplet. The change in the resistance value according to the number of printing times was smaller with $\mathrm{UV} / \mathrm{O}_{3}$ cleaning than without. The homogeneity of the thin film probably contributed to the smaller change in the resistance value. The homogeneity of the film increases the contact area between the PEDOT:PSS particles. Therefore, it is considered that the change in the resistance value with printing time become smaller.

Figure 5 shows the changes in visible light transmittance due to $\mathrm{UV} / \mathrm{O}_{3}$ cleaning and the number of printing times. Visible light transmittance decreased when $\mathrm{UV} / \mathrm{O}_{3}$ cleaning was performed. We assumed that when $\mathrm{UV} / \mathrm{O}_{3}$ cleaning was not performed on the substrate surface, ink could not be smoothly spread and gaps through which visible light passed were generated. By performing $\mathrm{UV} / \mathrm{O}_{3}$ cleaning, ink could be smoothly spread and a homogeneous thin film without gaps was formed; consequently, the transmittance decreased. However, the effect of $\mathrm{UV} / \mathrm{O}_{3}$ cleaning on the decrease in visible light transmittance was less than that of the number of printing times.

As mentioned above, by improving the state of the substrate surface, the number of printing times could be reduced while maintaining similar conductivity, and the decrease in transparency due to multiple times of printing could be inhibited.

\subsection{Removal of Additive by Annealing}

Figure 6 shows the change in the surface state of the thin film due to the annealing temperature. The surface state of the thin film became more heterogeneous as the annealing temperature increased. In the drying process, PEDOT:PSS particles can move and aggregate, resulting in an uneven surface. The evaporation rate of ethanol increased as the annealing temperature increased; consequently, this phenomenon was accelerated. The abovementioned aggregation might be the reason for the decreased visible light transmittance following the increased annealing temperature. It was presumed that 1$) \mathrm{UV} / \mathrm{O}_{3}$ cleaning of the substrate surface caused droplets to be evenly spread; 2) the aggregation of PEDOT:PSS particles occurred on the film surface with annealing; and 3) visible light was scattered and visible light transmittance decreased.

Figure 7 shows the change in the resistance value due to the annealing temperature and the number of printing times. The resistance value was lowest at an annealing temperature of $100^{\circ} \mathrm{C}$ with any number of printing times. Since the annealing temperature of $80^{\circ} \mathrm{C}$ was close to the boiling point of ethanol, there was a possibility that the additive did not evaporate completely and some remained in the thin film. The decrease in the resistance value was the least and its dispersion during measurement was the greatest when the annealing temperature was $150^{\circ} \mathrm{C}$. Because the boiling point of PEDOT:PSS is approximately $100^{\circ} \mathrm{C}$, a certain potion of PEDOT:PSS might have evaporated.

Figure 8 shows the change in visible light transmittance due to the annealing temperature and the number of printing times. Visible light transmittance was the highest (81\%) in a thin film with air drying and three printing times. Visible light transmittance decreased as the annealing temperature increased; however, the effect of annealing temperature on the decrease in visible light transmittance was less than that of the number of printing times.

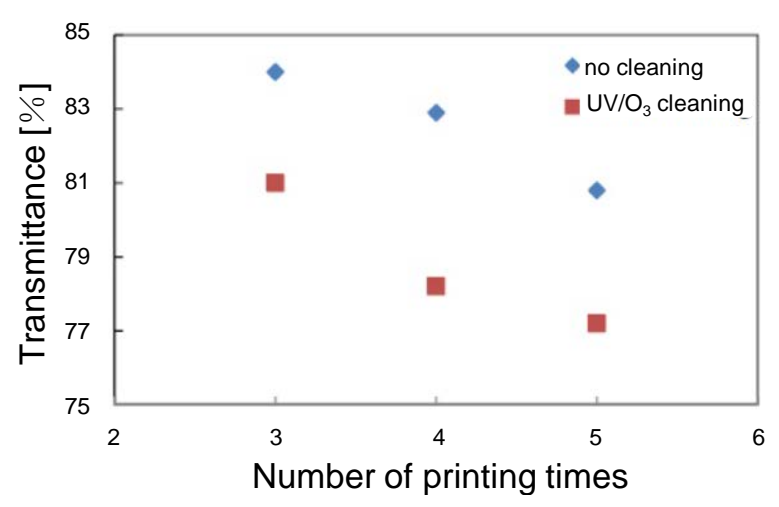

Figure 5. Dependence of the visible light transmittance due to $\mathrm{UV} / \mathrm{O}_{3}$ cleaning and the number of printing times. 


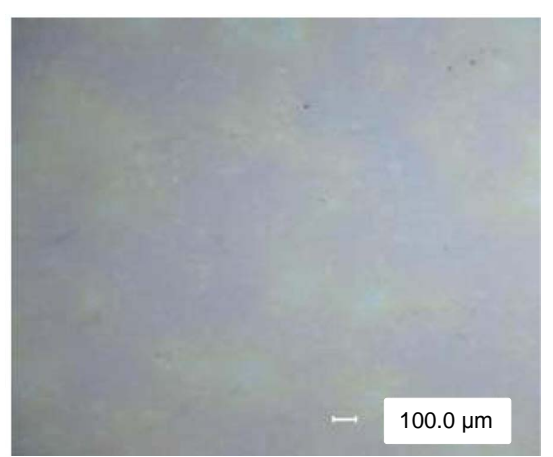

(a)

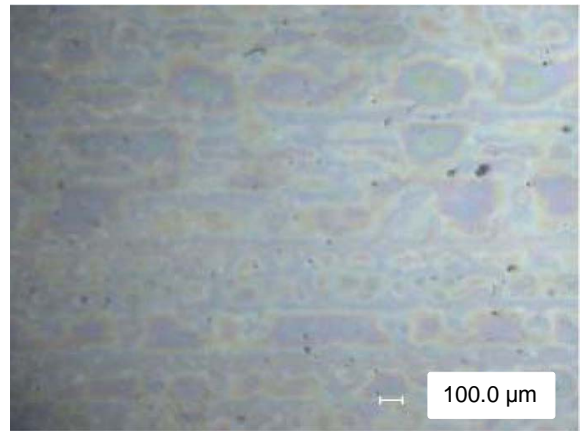

(c)

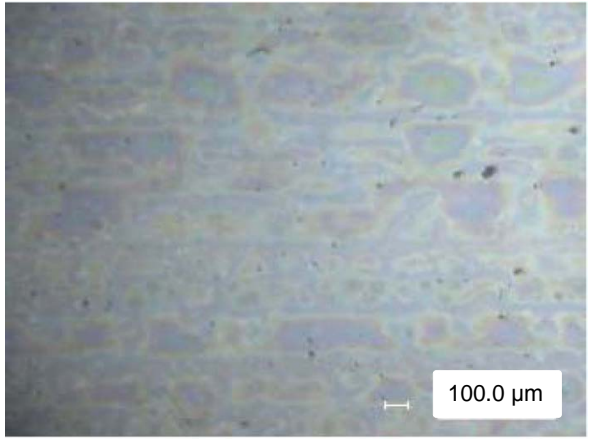

(b)

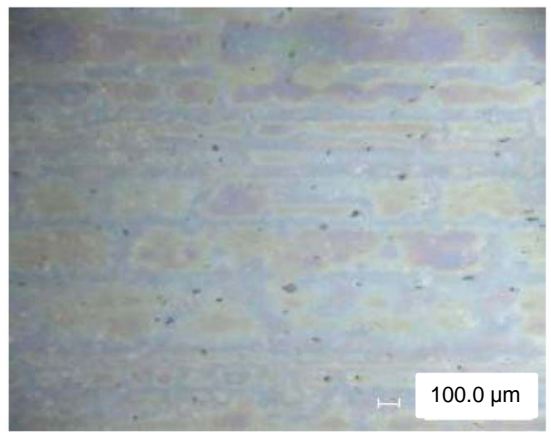

(d)

Figure 6. Microscope image of the surface of a PEDOT:PSS (3 times printing, $\times 100$ ). (a) No annealing; (b) $80^{\circ} \mathrm{C}$; (c) $100^{\circ} \mathrm{C}$; (d) $150^{\circ} \mathrm{C}$.

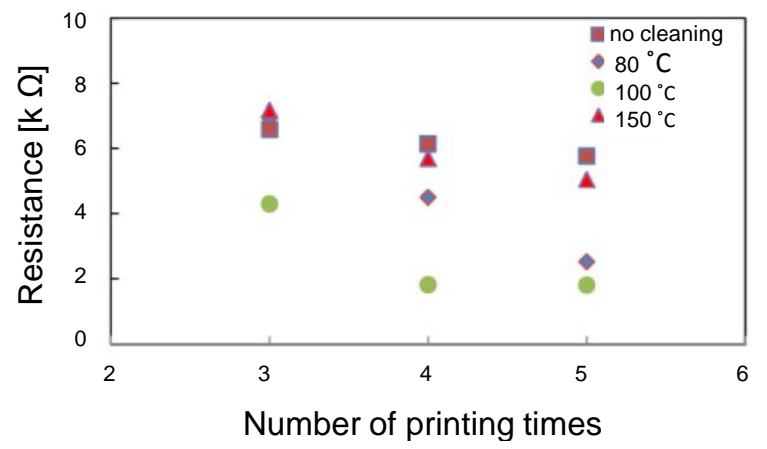

Figure 7. Dependence of the resistance value due to the annealing temperature and the number of printing times.

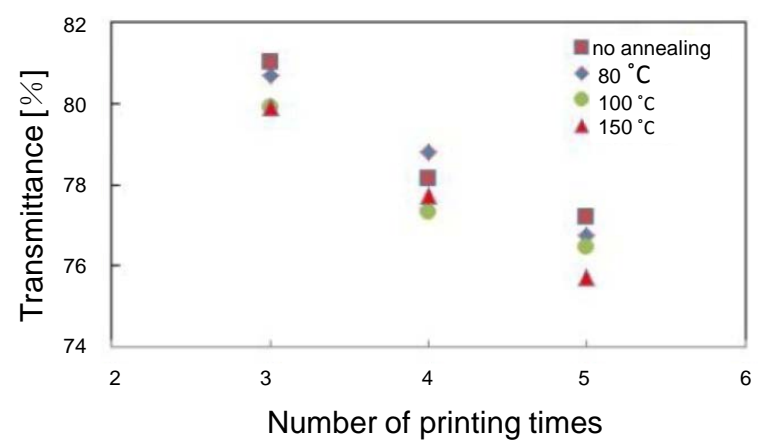

Figure 8. Dependence of the visible light transmittance due to the annealing temperature and the number of printing times. 
Although annealing after film deposition resulted in a decrease in visible light transmittance, the annealing was indispensable when the resistance value was taken into account. Since the effect of the number of printing times on the decrease in visible light transmittance was greater than that of the annealing temperature, reducing the number of printing times as much as possible while reducing the resistance value by annealing is essential.

\subsection{Dipping Treatment Using the Polar Solvent}

Figure 9 shows the change in the resistance value due to the dipping treatment and the number of printing times. The resistance value decreased when dipping treatment was performed. In particular, the dipping treatment had a large effect on a thin film formed by three printing times. With thin films formed by four and five printing times, the large amount of ink adhering to the thin film and the contact among PEDOT particles resulted in the dipping treatment having a small effect. There is a possibility that the dipping treatment with the polar solvent can reduce the number of printing times; consequently, a decrease in visible light transmittance due to multiple printing times can be inhibited.

Figure 10 shows the change in visible light transmittance due to the dipping treatment and the number of printing times. Figure 11 shows the change in the surface state of the thin film due to the dipping treatment. Visible light transmittance improved from the dipping treatment. Visible light transmittances of the thin films formed by three and four printing times were $83.3 \%$ and $82.0 \%$, respectively. Therefore, visible light transmittance above $80 \%$, which is required for a transparent conductive film, could be attained.

Aggregation of PEDOT:PSS particles due to the annealing heterogeneously occurred. However, with dipping treatment, the aggregation state became homogeneous. Because droplet sizes became similar to each other and the thin film surface became smooth with the dipping treatment, visible light transmittance increased. Thus, the dipping treatment after film deposition was revealed to be important to reconcile conductivity with transparency.

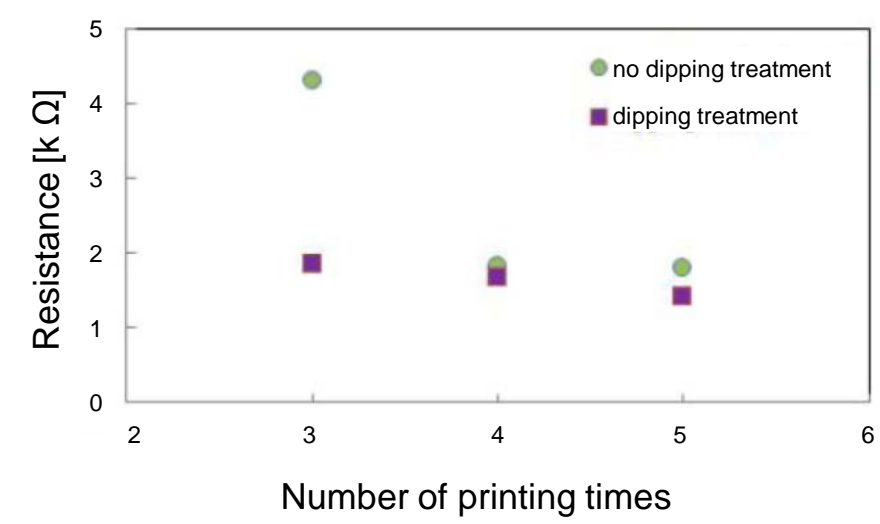

Figure 9. Dependence of the resistance value due to the dipping treatment and the number of printing times.

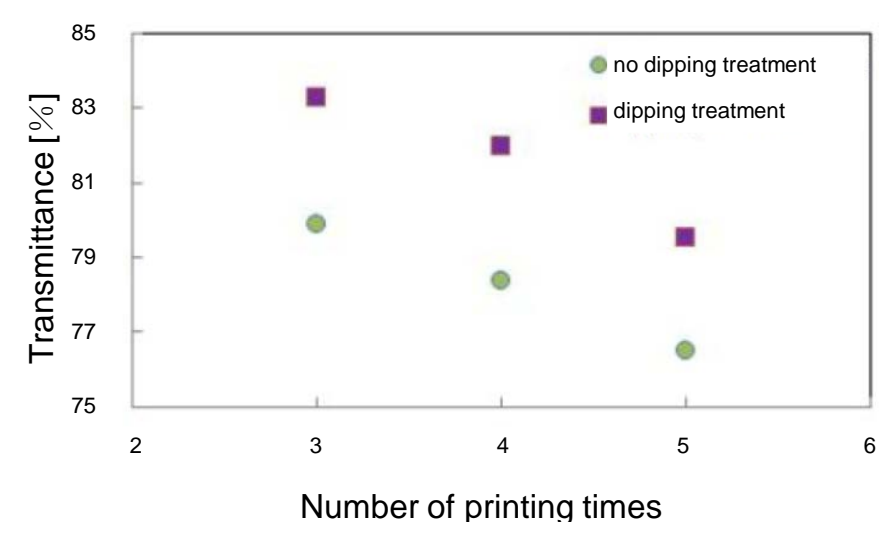

Figure 10. Dependence of the visible light transmittance due to the dipping treatment and the number of printing times. 


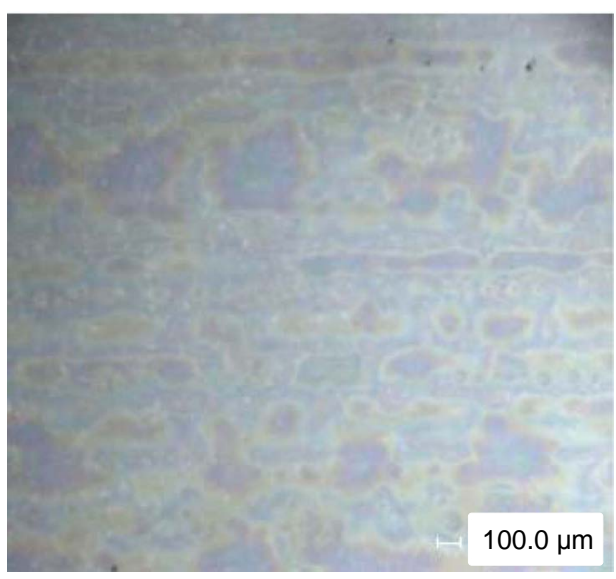

(a)

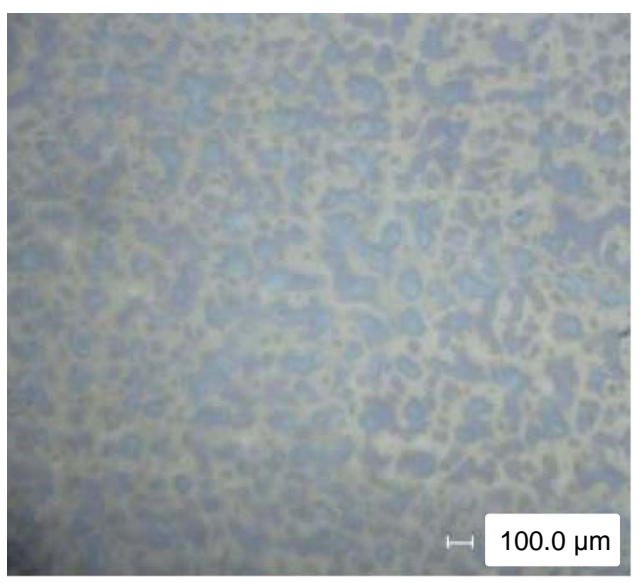

(b)

Figure 11. Microscope image of the surface of a PEDOT:PSS (annealing temperature: $100^{\circ} \mathrm{C}, 3$ times printing, $\times 100$ ). (a) Nodipping treatment; (b) Dipping treatment.

\section{Conclusions}

The present study's focus was to improve the characteristics of a PEDOT:PSS transparent conductive film, which was produced using an ink-jet printer. We printed several times in order to reduce the resistance value; consequently, the transparency worsened.

$\mathrm{UV} / \mathrm{O}_{3}$ cleaning of the surface of the film substrate resulted in a homogeneous thin film and a decrease in the resistance value. The resistance value decreased significantly when the number of printing times decreased. Therefore, the number of printing times can be reduced while maintaining the conductivity. In contrast, visible light transmittances decreased by $\mathrm{UV} / \mathrm{O}_{3}$ cleaning of the surface of the film substrate, probably because the ink could be spread smoothly and a homogeneous thin film without gaps could be formed. Consequently, visible light transmittances decreased.

The resistance value decreased by heating a thin film after it was formed. This is probably because the additive in the thin film evaporated and the amount of impurities decreased. However, droplets heterogeneously aggregated on the thin film surface. In the drying process, PEDOT:PSS particles might move and aggregate with each other, resulting in an uneven surface. The evaporation rate of ethanol increased as the annealing temperature increased; consequently, this phenomenon was accelerated, resulting in the scattering of visible light and the decrease in visible light transmittance.

The dipping treatment with the polar solvent caused the resistance value to decrease and visible light transmittance to improve. The dipping treatment had a large effect on the decrease in the resistance value of a thin film formed by three printing times. Reducing the number of printing times while maintaining similar conductivity is possible. The dipping treatment resulted in droplet sizes becoming similar to each other and a decrease in the amount of scattering light; consequently, visible light transmittance increased.

The present study revealed that the surface state of a film substrate, the annealing temperature after film deposition, and the dipping treatment using a polar solvent were related to the resistance value, surface state, and visible light transmittance of a thin film.

\section{References}

[1] Gasiorskia, P., Gondekb, E., Danelc, K.S., Pokladko-Kowarb, M., Armatysd, P. and Kityka, A.V. (2013) Green Electroluminescence from Azafluoranthene Derivatives in Polymer Based Electroluminescent Devices. Optical Materials, 35, 681-684. http://dx.doi.org/10.1016/j.optmat.2012.09.027

[2] Zhang, H., Xiao, J., Zeng, W. and Huang, W. (2014) Effect of PEDOT:PSS vs. $\mathrm{MoO}_{3}$ as the Hole Injection Layer on Performance of C545T-Based Green Electroluminescent Light-Emitting Diodes. Displays, 35, 171-175. http://dx.doi.org/10.1016/j.displa.2014.04.004

[3] Laura, C., Jose, A., Javier, P. and Antonio, U. (2015) Wavelength Influence on the Photodegradation of P3HT:PCBM Organic Solar Cells. Solar Energy Materials and Solar Cells, 141, 423-428. 
http://dx.doi.org/10.1016/j.solmat.2015.06.023

[4] Albrecht, G., Heuser, S., Keil, C. and Schlettwein, D. (2015) Strategy for Preparation of Transparent Organic Thin Film Transistors with PEDOT:PSS Electrodes and a Polymeric Gate Dielectric. Materials Science in Semiconductor Processing, 40, 772-776. http://dx.doi.org/10.1016/j.mssp.2015.07.057

[5] Wang, Z., Brown, E.S. and Maldonado, S. (2015) Hybrid Solar Cells Constructed of Macroporous N-Type GaP Coated with PEDOT:PSS. Chinese Chemical Letters, 26, 469-473. http://dx.doi.org/10.1016/j.cclet.2015.03.009

[6] Wegener, M., Eckert, D. and Roosen, A. (2015) Manufacture of Sub- $\mu \mathrm{m}$ Thin, Particulate-Based ITO Layers by Roller Coating. Journal of the European Ceramic Society, 35, 2321-2332. http://dx.doi.org/10.1016/j.jeurceramsoc.2015.02.012

[7] Kim, H.J., Seo, K.W., Kim, Y.H., Choi, J. and Kim, H.K. (2015) Direct Laser Patterning of Transparent ITO-Ag-ITO Multilayer Anodes for Organic Solar Cells. Applied Surface Science, 328, 215-221. http://dx.doi.org/10.1016/j.apsusc.2014.12.017

[8] Saghaei, J., Fallahzadeh, A. and Saghaei, T. (2015) ITO-Free Organic Solar Cells Using Highly Conductive PhenolTreated PEDOT:PSS Anodes. Organic Electronics, 24, 188-194. http://dx.doi.org/10.1016/j.orgel.2015.06.002

[9] Maniruzzaman, Md., Rahman, M.A., Jeong, K. and Lee, J. (2014) $\mathrm{MoO}_{3} / \mathrm{Au} / \mathrm{MoO}_{3}$-PEDOT:PSS Multilayer Electrodes for ITO-Free Organic Solar Cells. Materials Science in Semiconductor Processing, 27, 114-120. http://dx.doi.org/10.1016/j.mssp.2014.06.034

[10] Miura, S., Tashiro, M., Suzuki, K., Noda, S., Hori, S. and Nonomura, S. (2015) Stacked Nanoparticle-Transparent Conductive Oxide Substrate Combining High Haze with Low Surface Roughness for Improvement of Thin Film Si Solar-Cell Performance. Thin Solid Films, 574, 78-83. http://dx.doi.org/10.1016/j.tsf.2014.11.086

[11] Tashkhourian, J., Hemmateenejad, B., Beigizadeh, H., Hosseini-Sarvari, M. and Razmi, Z. (2014) ZnO Nanoparticles and Multiwalled Carbon Nanotubes Modified Carbon Paste Electrode for Determination of Naproxen Using Electrochemical Techniques. Journal of Electroanalytical Chemistry, 714-715, 103-108. http://dx.doi.org/10.1016/j.jelechem.2013.12.026

[12] Han, S.H., Kim, B.J. and Park, J.S. (2014) Effects of the Corona Pretreatment of PET Substrates on the Properties of Flexible Transparent CNT Electrodes. Thin Solid Films, 572, 73-78. http://dx.doi.org/10.1016/j.tsf.2014.09.066

[13] Kim, B.J., Han, S.H. and Park, J.S. (2015) Properties of CNTs Coated by PEDOT:PSS Films via Spin-Coating and Electrophoretic Deposition Methods for Flexible Transparent Electrodes. Surface and Coatings Technology, 271, 2226. http://dx.doi.org/10.1016/j.surfcoat.2015.01.045

[14] Orgiu, E., Manunza, I., Sanna, M., Cosseddu, P. and Bonfiglio, A. (2008) Transparent Dielectric Films for Organic Thin-Film Transistors: A Perspective for Low Cost, Low Size Technologies. Thin Solid Films, 516, 1533-1537. http://dx.doi.org/10.1016/j.tsf.2007.03.157

[15] Kim, I., Kwak, S.W., Ju, Y., Park, G.Y., Lee, T.M., Jang, Y., Choi, Y.M. and Kang, D. (2015) Roll-Offset Printed Transparent Conducting Electrode for Organic Solar Cells. Thin Solid Films, 580, 21-28. http://dx.doi.org/10.1016/j.tsf.2015.02.075

[16] Hübler, A.C., Schmidt, G.C., Kempa, H., Reuter, K., Hambsch, M. and Bellmann, M. (2011) Three-Dimensional Integrated Circuit Using Printed Electronics. Organic Electronics, 12, 419-423. http://dx.doi.org/10.1016/j.orgel.2010.12.010

[17] Willmann, J., Stocker, D. and Dörsam, E. (2014) Characteristics and Evaluation Criteria of Substrate-Based Manufacturing. Is Roll-to-Roll the Best Solution for Printed Electronics? Organic Electronics, 15, 1631-1640. http://dx.doi.org/10.1016/j.orgel.2014.04.022

[18] Zhang, Z. and Zhu, W. (2015) Controllable Synthesis and Sintering of Silver Nanoparticles for Inkjet-Printed Flexible Electronics. Journal of Alloys and Compounds, 649, 687-693. http://dx.doi.org/10.1016/j.jallcom.2015.07.195

[19] Ali, S., Bae, J., Lee, C.H., Choi, K.H. and Doh, Y.H. (2015) All-Printed and Highly Stable Organic Resistive Switching Device Based on Graphene Quantum Dots and Polyvinylpyrrolidone Composite. Organic Electronics, 25, 225-231. http://dx.doi.org/10.1016/j.orgel.2015.06.040

[20] Castro, H.F., Sowade, E., Rocha, J.G., Alpuim, P., Machado, A.V., Baumann, R.R. and Lanceros-Méndez, S. (2015) Degradation of All-Inkjet-Printed Organic Thin-Film Transistors with TIPS-Pentacene under Processes Applied in Textile Manufacturing. Organic Electronics, 22, 12-19. http://dx.doi.org/10.1016/j.orgel.2015.03.028

[21] Jönsson, S.K.M., Birgerson, J., Crispin, X., Greczynski, G., Osikowicz, W., Denier van der Gon, A.W., Salaneck, W.R. and Fahlman, M. (2003) The Effects of Solvents on the Morphology and Sheet Resistance in Poly(3,4-ethylenedioxythiophene)-Polystyrenesulfonic Acid (PEDOT-PSS) Films. Synthetic Metals, 139, 1-10. http://dx.doi.org/10.1016/S0379-6779(02)01259-6

[22] Yan, H. and Okuzaki, H. (2009) Effect of Solvent on PEDOT/PSS Nanometer-Scaled Thin Films: XPS and STEM/ AFM Studies. Synthetic Metals, 159, 2225-2228. http://dx.doi.org/10.1016/j.synthmet.2009.07.032 
[23] Shimono, K. and Nitta, A. (2013) A Study of Transparent Conductive Film by Inkjet Printing. The 66th Joint Conference of Electrical and Electronics Engineers in Kyushu, Kumamoto, 24-25 September 2013, 285.

[24] Takano, T., Masunaga, H., Fujiwara, A., Okuzaki, H. and Sasaki, T. (2012) PEDOT Nanocrystal in Highly Conductive PEDOT:PSS Polymer Films. Macromolecules, 45, 3859-3865. http://dx.doi.org/10.1021/ma300120g 